\title{
Confinement of magnetic vortex and domain walls in dipolar coupled concentric nanocylinders
}

Conference Paper · May 2015

DOI: 10.1109/INTMAG.2015.7156968

CITATIONS

0

6 authors, including:
READS

10

\section{Ana L. Dantas}

Universidade do Estado do Rio Grande do Nort... 53 PUBLICATIONS 284 CITATIONS

SEE PROFILE

\section{Artur Carriço}

Universidade Federal do Rio Grande do Norte 85 PUBLICATIONS 630 CITATIONS

SEE PROFILE

Some of the authors of this publication are also working on these related projects: 


\title{
Confinement of Magnetic Vortex and Domain Walls in Dipolar-Coupled Concentric Nanocylinders
}

\author{
Leonardo L. Oliveira ${ }^{1}$, Marcos S. Nunes ${ }^{2}$, Claudivan M. Souza ${ }^{1}$, Ana L. Dantas ${ }^{2}$, \\ Idalmir Q. Souza, Jr. ${ }^{3}$, Gustavo O. G. Rebouças ${ }^{3}$, and A. S. Carriço ${ }^{1}$ \\ ${ }^{1}$ Department of Physics, University of Rio Grande do Norte, Natal 59078-900, Brazil \\ ${ }^{2}$ Department of Physics, Universidade do Estado do Rio Grande do Norte, Mossoró 59900-000, Brazil \\ ${ }^{3}$ Department of Physics, Universidade Federal Rural do Semi-Árido, Mossoró 59625-900, Brazil
}

\begin{abstract}
We report a theoretical study of the magnetic phases of core-shell nanocylinders, consisting of a Py cylindrical core, dipolar coupled to a coaxial Fe cylindrical shell. A few nanometers thick nonmagnetic cylindrical layer separates the core from the shell, and controls the magnitude of the core-shell dipolar interaction. New magnetic phases emerge from the dipolar interaction, and may consist of either the combination of the intrinsic magnetic phases or new phases that are not seen in isolated cylinders and shells. We discuss typical examples. The magnetic phases of a $21 \mathrm{~nm}$-height nanocylinder composed of a 57 nm-diameter Py core coupled to a $12 \mathrm{~nm}$-thick Fe shell may be set to be a Py vortex with the same chirality of the Fe shell circular state, or a Py uniform domain coupled to a pair of domain walls of the Fe shell onion state. A magnetic vortex may be stabilized in a $6 \mathrm{~nm}$-height, $42 \mathrm{~nm}$-diameter Py cylinder coupled to a $6 \mathbf{n m}$-thick Fe shell.
\end{abstract}

Index Terms-Bimagnetic core-shell, dipolar interaction, domain walls, magnetic vortex.

\section{INTRODUCTION}

$\mathbf{F}$ ERROMAGNETIC nanostructures have recently attracted an extensive research effort. The interest stems from both the chances of designing new systems for fundamental studies of magnetism in confined geometries, and from the promising perspectives of a variety of emerging applications, such as spin logic devices, magnetic sensors, magnetic nano-oscillators, and magnetic memories [1]-[5]. Vortices and domain walls are issues of current interest in these systems. Key applications benefit from the possibility of tailoring the vortex core and the domain-wall magnetic patterns.

Spherical bimagnetic core-shell nanoparticles systems have been investigated for applications, such as systems for permanent magnets, recording media, and magnetic hyperthermia, exploring the possibility of combining different functionalities of two magnetic materials into the effective magnetic properties of a single nanoparticle [6].

We presently report a theoretical study of the magnetic phases of cylindrical core-shells, consisting of a Py cylindrical core and an Fe coaxial cylindrical shell, separated by a few nanometers thick nonmagnetic cylindrical layer.

Cylindrical core-shells comprise two elements, which are currently the focus of extensive investigations: 1) the magnetic vortex, which may nucleate in the core cylinder, and which holds great promise as the key element of a new generation of microwave nano-oscillators [1], [2] and 2) the onion and vortex states of ferromagnetic rings, which are currently being considered as a possible new route to the design of magnetic memories [3]-[5].

We show that the core-shell dipolar coupling has a relevant impact in the magnetic phases, affecting both the nucleation

Manuscript received March 20, 2015; revised June 17, 2015; accepted July 13, 2015. Date of publication July 28, 2015; date of current version October 22, 2015. Corresponding author: A. L. Dantas (e-mail: dantasal@gmail.com).

Color versions of one or more of the figures in this paper are available online at http://ieeexplore.ieee.org.

Digital Object Identifier 10.1109/TMAG.2015.2459033

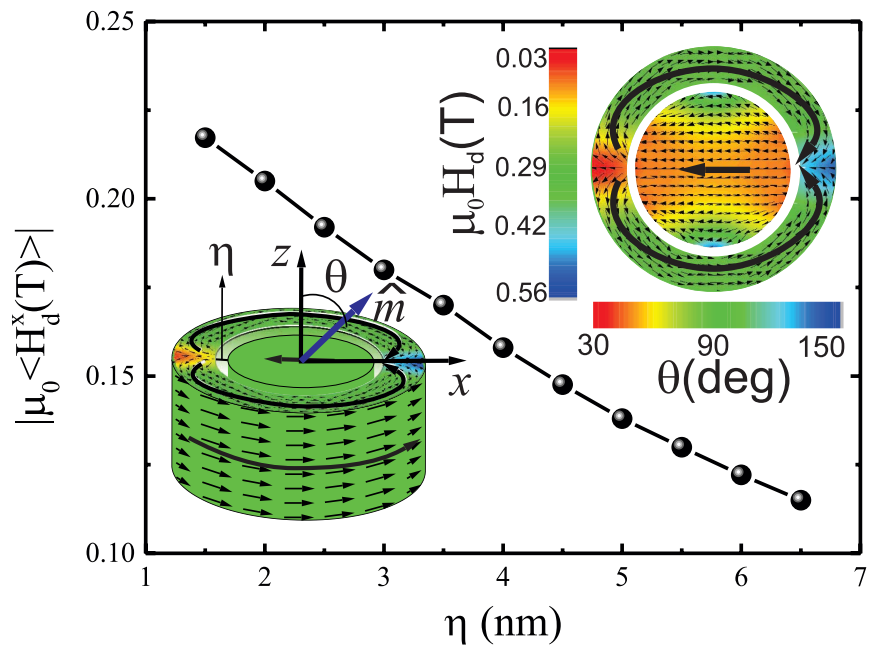

Fig. 1. Dipolar field produced by the $12 \mathrm{~nm}$-thick Fe shell averaged over the whole Py cylinder, in a $21 \mathrm{~nm}$-height $\mathrm{Py}(57 \mathrm{~nm}) / \eta \mathrm{nm} / \mathrm{Fe}(12 \mathrm{~nm})$ core-shell. Top inset: dipolar field produced by the $12 \mathrm{~nm}$-thick Fe shell on the Py cylinder and the spin map of the onion state in the Fe shell, within the top layer of the core-shell, for $\eta=3 \mathrm{~nm}$. Left color bar codes: dipolar field strength, in $\mathrm{T}$, in the top layer of the Py cylinder. Bottom color bar code: out-of-plane angle in the spin map within the Fe shell. Bottom inset: Fe domain walls pinned at the poles of the Py cylinder. $\hat{m}$ : magnetic moment direction.

of a magnetic vortex in the core cylinder and the position of domain-wall pairs in the cylindrical shell.

By choosing the nonmagnetic layer thickness $[\eta$ shown in Fig. 1 (bottom inset)], one may inhibit the formation of a vortex in the Py cylinder, favoring the formation of a uniform state in the Py inner cylinder, dipolar coupled to an onion state in the Fe shell. The core-shell dipolar interaction pins the head-to-head and tail-to-tail domain walls of the Fe shell near the magnetic poles of the inner cylinder. This is shown in Fig. 1 for a $21 \mathrm{~nm}$-height $(h) \operatorname{Py}(57 \mathrm{~nm}) / 3 \mathrm{~nm} / \mathrm{Fe}(12 \mathrm{~nm})$ core-shell, consisting of a $57 \mathrm{~nm}$-diameter Py cylinder and a $12 \mathrm{~nm}$-thick Fe shell, separated by a $3 \mathrm{~nm}$-thick nonmagnetic layer. 

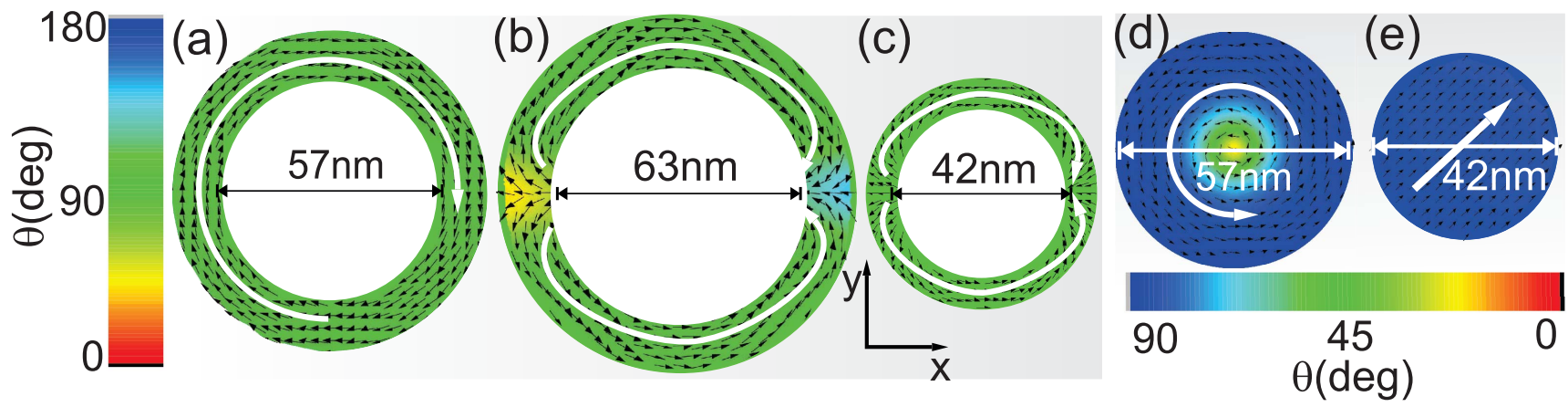

Fig. 2. Spin maps for the magnetic phases of isolated Fe shells and Py cylinders. (a) Vortex state of a $21 \mathrm{~nm}$-height and $12 \mathrm{~nm}$-thick Fe shell with an inner diameter of $57 \mathrm{~nm}$. (b) Onion state of a $21 \mathrm{~nm}$-height and $12 \mathrm{~nm}$-thick Fe shell with an inner diameter of $63 \mathrm{~nm}$. (c) Onion state of a $6 \mathrm{~nm}$-height, $6 \mathrm{~nm}$-thick Fe shell with a $42 \mathrm{~nm}$ inner diameter. (d) Vortex state of a $21 \mathrm{~nm}$-height, $57 \mathrm{~nm}$-diameter Py cylinder. (e) Uniform state of a $6 \mathrm{~nm}$-height, $42 \mathrm{~nm}$-diameter Py cylinder. Left color bar code: out-of-plane angle in the spin map within the Fe shells. Bottom color bar code: out-of-plane angle in the spin map within the Py cylinders.

More interestingly, we also show that the core-shell dipolar interaction may lead to a relevant reduction of the threshold geometrical dimensions, for vortex nucleation in the cylindrical core.

\section{THEORETICAL MODEL}

The magnetic structure is described using small cubic cells, with edge smaller than the domain-wall width and exchange lengths of the core and shell materials. The $x$-axis is chosen along the uniaxial anisotropy of the Fe shell. The energy density is given by

$$
\begin{aligned}
E= & -\vec{H} \cdot \sum_{j} M_{S}^{j} \hat{m}_{j}-\sum_{j} K^{j}\left(m_{j}^{x}\right)^{2} \\
& +\frac{1}{2} \sum_{j} \sum_{k} M_{S}^{j} M_{S}^{k}\left(\frac{\hat{m}_{j} \cdot \hat{m}_{k}}{n_{j k}^{3}}-\frac{3\left(\hat{m}_{j} \cdot \hat{n}_{j k}\right)\left(\hat{m}_{k} \cdot \hat{n}_{j k}\right)}{n_{j k}^{5}}\right) \\
& +\frac{1}{d^{2}} \sum_{j} \sum_{k} A_{j k}\left(1-\hat{m}_{j} \cdot \hat{m}_{k}\right) .
\end{aligned}
$$

The first two terms are the Zeeman and anisotropy energies, and $M_{S}^{j}$ and $\hat{m}_{j}$ are the $j$-cell saturation magnetization value and direction, respectively. In the dipolar energy, $n_{j k}$ is the distance between the cells $j$ and $k$ in units of cell edge. The exchange energy couples nearest neighbor cells. $A_{j k}$ is either the core or the shell exchange stiffness, for cells $j$ and $k$ within the same material. We neglect possible exchange energy between the core and the shell.

The equilibrium configuration is found using numeric procedures that allow finding the magnetic patterns in a selfconsistent manner [8]. At each cell, the magnetization direction $\hat{m}_{j}$ is adjusted to be parallel to the local magnetic field $\left[H_{\text {eff }}^{j}=-\left(1 / M_{S}\right)\left(\partial E / \partial \hat{m}_{j}\right)\right]$, so that, within a reasonable numerical precision, the torque is zero, $\vec{m}_{j} \times \vec{H}_{\text {eff }}^{j}=0$. Convergence is checked to guarantee a maximum torque of $10^{-26} \mathrm{~J}$.

For iron, we use $M_{S}=1.7 \times 10^{6} \mathrm{~A} / \mathrm{m}$, exchange stiffness $A=2.5 \times 10^{-11} \mathrm{~J} / \mathrm{m}$, and anisotropy energy density $K=4.7 \times 10^{4} \mathrm{~J} / \mathrm{m}^{3}$ [9]. For Permalloy, we use $M_{S}=0.8 \times 10^{6} \mathrm{~A} / \mathrm{m}, A=1.3 \times 10^{-11} \mathrm{~J} / \mathrm{m}$, and $K=0$. We use $d=3 \mathrm{~nm}$ for $21 \mathrm{~nm}$-height nanocylinders and cylindrical core-shells, and $d=2 \mathrm{~nm}$ for $6 \mathrm{~nm}$-height nanocylinders and cylindrical core-shells. In both cases, $d$ is much smaller than the exchange length of Fe and Py.
The magnetic phases are calculated starting with a large value of the external field strength to achieve the saturation of the magnetization along the external field direction. The remnant magnetic phase is obtained by reducing the external field strength down to $H=0$. For each value of the external field, the self-consistent procedure is initialized with the magnetic state corresponding to the equilibrium state of the previous value of the external field.

We use two preparation routes to obtain the remnant phase. We either saturate the system using a large external field along the Fe anisotropy direction or along the $z$-axis direction, perpendicular to the core-shell surface.

The sequence of magnetic phases and the remnant phase may be different for the $z$-axis and $x$-axis routes, due to the intrinsic properties of the core and shell systems, as well as to the core-shell dipolar coupling.

\section{RESULTS AND DISCUSSION}

Before discussing the core-shell magnetic phases, it is instructive to review some of the key magnetic phases of isolated Py cylinders and Fe cylindrical shells (rings). Fig. 2 shows our results for some of the magnetic configurations of Py cylinders and Fe shells that compose the core-shell magnetic phases.

Thin cylindrical shells exhibit two interesting and, to some extent, simple equilibrium states at remanence: 1) the vortex and 2) the onion states. The vortex state [7] corresponds to a state of circular magnetization, similar to the vortex state of nanocylinders, but lacking a vortex core. The onion state [4] is composed of two parts with circulation in the opposite directions, separated by a domain-wall pair: 1) a head-to-head domain wall at one end and 2) a tail-to-tail domain at the other end to grant opposite circulation in the two branches of the onion state.

Our results for the magnetic phases at $H=0$ are summarized in Table I. We have found that thin Fe shells, with dimensions in the sub-100 $\mathrm{nm}$ range, tend to form the vortex state, for small values of the inner diameter. The onion state is favored for larger values of the inner diameter.

Fig. 2(a) shows the vortex state of a $21 \mathrm{~nm}$-height, $12 \mathrm{~nm}$-thick Fe cylindrical shell with a $57 \mathrm{~nm}$ inner diameter. Fig. 2(b) shows the onion state of a $21 \mathrm{~nm}$-height, $12 \mathrm{~nm}$-thick $\mathrm{Fe}$ cylindrical shell with a $63 \mathrm{~nm}$ inner diameter. 
TABLE I

Magnetic Phases of Py-Fe Cylindrical Core-Shells

\begin{tabular}{|c|c|c|}
\hline$\eta(\mathrm{nm})$ & $\begin{array}{c}\mathrm{Py}(57 \mathrm{~nm}) / \eta(\mathrm{nm}) / \mathrm{Fe}(12 \mathrm{~nm}) \\
h=21 \mathrm{~nm} \\
H_{x}(x-\text { axis route })\end{array}$ & $\begin{array}{c}\text { Isolated } \mathrm{Fe}(12 \mathrm{~nm}) \text { shell } \\
\text { inner diameter } \\
(57 \mathrm{~nm}+2 \eta)\end{array}$ \\
\hline $0.0-1.0 \mathrm{~nm}$ & Py vortex and $\mathrm{Fe}$ vortex state & vortex state \\
\hline $1.5-6.5 \mathrm{~nm}$ & Py uniform and $\mathrm{Fe}$ onion state & onion state \\
\hline$>7.0 \mathrm{~nm}$ & Py vortex and $\mathrm{Fe}$ onion state & onion state \\
\hline$\eta(\mathrm{nm})$ & $\begin{array}{c}\mathrm{Py}(42 \mathrm{~nm}) / \eta(\mathrm{nm}) / \mathrm{Fe}(6 \mathrm{~nm}) \\
h=6 \mathrm{~nm} \\
H_{z}(z-\text { route })\end{array}$ & $\begin{array}{l}\text { Isolated } \mathrm{Fe}(6 \mathrm{~nm}) \text { shell } \\
\text { inner diameter } \\
(42 \mathrm{~nm}+2 \eta)\end{array}$ \\
\hline $0.0-1.2 \mathrm{~nm}$ & Py vortex and $\mathrm{Fe}$ vortex state & onion state \\
\hline $1.4-2.0 \mathrm{~nm}$ & Py uniform and $\mathrm{Fe}$ vortex state & onion state \\
\hline$>2.4 \mathrm{~nm}$ & Py uniform and $\mathrm{Fe}$ onion state & onion state \\
\hline
\end{tabular}

Fig. 2(c) shows the onion state of a $6 \mathrm{~nm}$-height, $6 \mathrm{~nm}$-thick Fe cylindrical shell with $42 \mathrm{~nm}$ inner diameter.

Fig. 2(d) shows the vortex state of a $21 \mathrm{~nm}$-height, $57 \mathrm{~nm}$-diameter Py cylinder. Fig. 2(e) shows the uniform state of a $6 \mathrm{~nm}$-height, $42 \mathrm{~nm}$-diameter Py disk.

Using the $x$-axis route, we have found that the core-shell magnetic phases are largely controlled by the magnetic phases of the Fe shell. This sounds intuitive since Fe has larger values of the magnetization, exchange, and anisotropy energies. The balance of intrinsic $\mathrm{Fe}$ exchange, dipolar and anisotropy energies, subjected to the nanometer scale geometrical constrictions, controls the magnetic phases of isolated Fe shells. The Py cylinder dipolar field produces no relevant changes in this picture, as shown in Table I.

For the present discussion, we have chosen two core-shell configurations: 1) a $21 \mathrm{~nm}$-height $\mathrm{Py}(57 \mathrm{~nm}) / \eta(\mathrm{nm}) / \mathrm{Fe}(12 \mathrm{~nm})$ and 2) a $6 \mathrm{~nm}$-height $\mathrm{Py}(42 \mathrm{~nm}) / \eta(\mathrm{nm}) / \mathrm{Fe}(6 \mathrm{~nm})$.

We have used the $x$-axis route to investigate the magnetic phases of the $\mathrm{Py}(57 \mathrm{~nm}) / \eta(\mathrm{nm}) / \mathrm{Fe}(12 \mathrm{~nm})$ core-shell. The reason being that the $21 \mathrm{~nm}$-height, $57 \mathrm{~nm}$-diameter Py-isolated cylinder holds a vortex at remanence, as shown in Fig. 2, using either the $x$-axis route or the $z$-axis route. On the other hand, using the $x$-axis route, we have found that $21 \mathrm{~nm}$-height isolated $\mathrm{Fe}$ shells hold a vortex state at remanence, for inner diameter values ranging from 57 up to $59 \mathrm{~nm}$. For larger values of the inner diameter, the onion state is the magnetic phase at remanence.

We show that by choosing the value of the nonmagnetic spacer layer, using the $x$-axis route, we may either favor the vortex formation in the Py cylinder and in the Fe shell or one may favor the formation of the onion state in the Fe shell and the uniform state in the Py cylinder.

As shown in Table I, the Py vortex is kept in the core-shell if the Fe shell inner diameter is $<60 \mathrm{~nm}$. However, for larger values of the inner $\mathrm{Fe}$ shell diameter, the core-shell consists of a uniformly magnetized Py cylinder, dipolar coupled with the pair of domain walls of the Fe shell.

As shown in Fig. 2, a 21 nm-height, 57 nm-diameter Py-isolated cylinder holds a vortex with a core diameter of $36 \mathrm{~nm}$. However, as shown in Fig. 1, the magnetic phase of a $21 \mathrm{~nm}$-height $\mathrm{Py}(57 \mathrm{~nm}) / 3 \mathrm{~nm} / \mathrm{Fe}(12 \mathrm{~nm})$ core-shell comprises a single-domain Py cylinder dipolar coupled to the Fe onion state, with the Fe domain walls located at the poles of the inner cylinder.

This arrangement of core-shell magnetization favors the core-shell dipolar interaction. As shown in Fig. 1 (inset), the $\mathrm{Fe}$ shell produces a dipolar field in the inner cylinder,

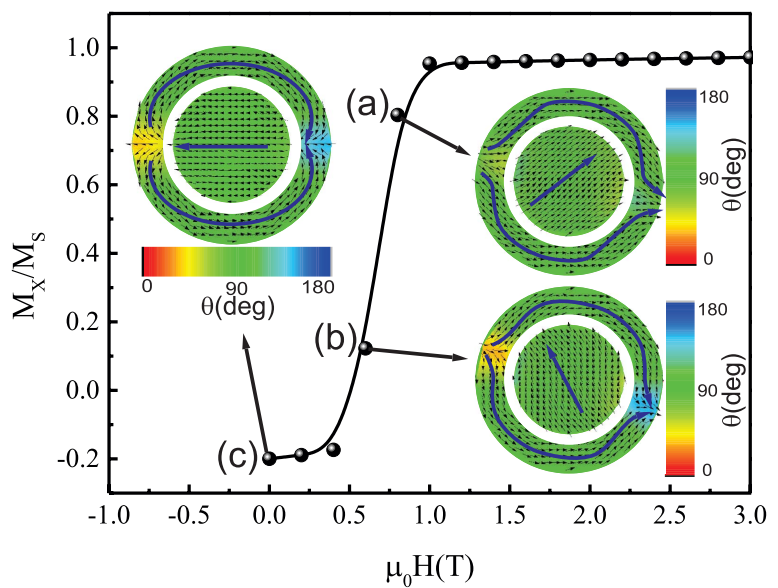

Fig. 3. Magnetization curve for the external field along the $x$-axis route (along the Fe shell anisotropy field) for a $21 \mathrm{~nm}$-height $\operatorname{Py}(57 \mathrm{~nm}) / 3 \mathrm{~nm} / \mathrm{Fe}(12 \mathrm{~nm})$ core-shell. Insets: magnetization profiles at selected points of the magnetization curve, for the external field strength of (a) 0.8 , (b) 0.6 , and (c) $0 \mathrm{~T}$. Color bar codes: out-of-plane angle in the spin map.

which is along the direction connecting the Fe shell domain walls, with strength ranging from 0.03 up to $0.56 \mathrm{~T}$, and an average value of $0.18 \mathrm{~T}$.

In Fig. 1, we also show the average value, in the volume of the Py cylinder, of the dipolar field produced by the domainwall pair of the $\mathrm{Fe}$ shell onion state. Notice that the average dipolar field drops from $0.22 \mathrm{~T}$, for $\eta=1.5 \mathrm{~nm}$ (corresponding to an $\mathrm{Fe}$ shell inner diameter of $60 \mathrm{~nm}$ ), down to $0.11 \mathrm{~T}$ for $\eta=6.5 \mathrm{~nm}$ (corresponding to an Fe shell inner diameter of $70 \mathrm{~nm}$ ). For larger values of $\eta$, the influence of the Fe shell dipolar field is no longer enough to inhibit the magnetic vortex in the Py cylinder.

For all cases investigated, a $0.5 \mathrm{~T}$ external field is enough for saturation along the external field direction ( $x$-axis direction). Furthermore, as shown in Fig. 3, the magnetic phases are tunable by the external field strengths smaller than $0.1 \mathrm{~T}$. This may turn to be interesting for the ring memory design, for instance, because the position of the Fe shell domain walls can be controlled using the modest external field values. As shown in Fig. 3, the sequence of magnetic states of a $21 \mathrm{~nm}$-height $\operatorname{Py}(57 \mathrm{~nm}) / 3 \mathrm{~nm} / \mathrm{Fe}(12 \mathrm{~nm})$ core-shell shows a fine-tuning of the orientation of the Py magnetization and the position of the Fe shell domain-wall pair in an external field interval of $0.1 \mathrm{~T}$.

More striking, as shown in Fig. 4, using the $z$-axis field route, one may induce vortex nucleation in a $6 \mathrm{~nm}$-height, $42 \mathrm{~nm}$-diameter Py core cylinder, dipolar coupled to a $6 \mathrm{~nm}$-width $\mathrm{Fe}$ shell. This sounds surprising since a $42 \mathrm{~nm}$-diameter Py cylinder requires a $12 \mathrm{~nm}$ minimum height to hold a vortex.

As shown in Fig. 2, a $6 \mathrm{~nm}$-height, $42 \mathrm{~nm}$-diameter Py cylinder has a uniform in-plane state. However, as shown in Table I, we have found that $6 \mathrm{~nm}$-height Py/Fe core-shells, with the nonmagnetic layer thickness ranging from 0 up to $1.2 \mathrm{~nm}$, the Py cylinder exhibit a magnetic vortex, coupled to Fe shell vortex with the same chirality. The Py cylinder vortex spreads over most of the Py core area, with a vortex core diameter of $32 \mathrm{~nm}$.

The dipolar field has a relevant impact on the sequence of phases. By reducing the external field strength from 

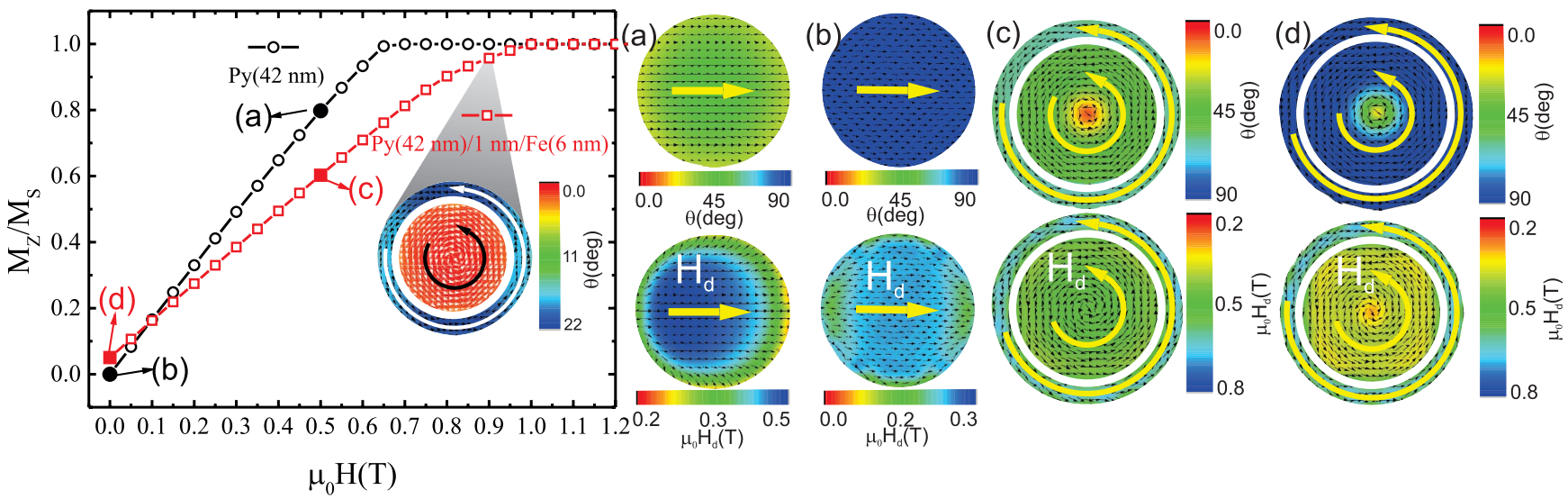

Fig. 4. Magnetization curves for the external field along the $z$-axis route. Square (circle) symbols: $6 \mathrm{~nm}$-height Py $(42 \mathrm{~nm}) / 1 \mathrm{~nm} / \mathrm{Fe}(6 \mathrm{~nm})$ core-shell [isolated $6 \mathrm{~nm}$-height $\mathrm{Py}(42 \mathrm{~nm})$ cylinder]. Inset: spin map, at $\mu_{0} H=0.95 \mathrm{~T}$, for the $6 \mathrm{~nm}$-height Py $(42 \mathrm{~nm}) / 1 \mathrm{~nm} / \mathrm{Fe}(6 \mathrm{~nm})$ core-shell. Color bar code: out-of-plane angle. Right: magnetization profile in the top row and the dipolar field in the bottom row for selected points. (a) For $\mu_{0} H=0.5 \mathrm{~T}$ and (b) $H=0$, for isolated $6 \mathrm{~nm}$ height $\mathrm{Py}(42 \mathrm{~nm})$ cylinder. (c) For $\mu_{0} H=0.5 \mathrm{~T}$ and (d) $H=0$, for $6 \mathrm{~nm}$ height Py $(42 \mathrm{~nm}) / 1 \mathrm{~nm} / \mathrm{Fe}(6 \mathrm{~nm})$ core-shell. Color bar codes: out-of-plane angle in the top row and the strength (in T) of the dipolar field in the bottom row. In the inset and in the right panels, the spin and dipolar field maps show the projection in the $x y$ plane.

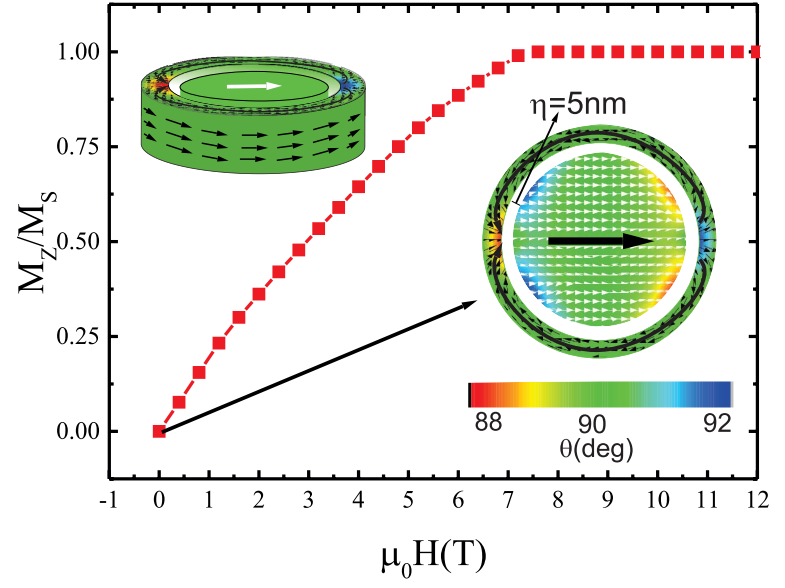

Fig. 5. Magnetization curve for the external field along the $z$-axis route for a $6 \mathrm{~nm}$-height $\mathrm{Py}(42 \mathrm{~nm}) / 5 \mathrm{~nm} / \mathrm{Fe}(6 \mathrm{~nm})$ core-shell. Right inset: magnetization profile for $H=0$, projected in the $x y$ plane. Left inset: corresponding 3-D picture of the $\mathrm{Fe}$ domain walls pinned at the poles of the Py cylinder. Color bar codes: out-of-plane angle in both panels.

the saturation, the magnetization of the $6 \mathrm{~nm}$-height $\operatorname{Py}(6 \mathrm{~nm}) / 1 \mathrm{~nm} / \mathrm{Fe}(6 \mathrm{~nm})$ core-shell drops faster than that of $6 \mathrm{~nm}$-height $\mathrm{Py}(42 \mathrm{~nm})$ cylinder. At $\mu_{0} H_{z}=0.95 \mathrm{~T}$, a spiral state forms in the Py cylinder. This is a vortexlike state, as shown in Fig. 4 (inset), with the large value of $M_{z}\left(M_{z}=0.98 M_{S}\right)$. As $H$ is reduced, this state evolves to the vortex state found for $H=0$, and drives the Fe shell together in a vortex state.

We have also found that a $6 \mathrm{~nm}$-height, $56 \mathrm{~nm}$-diameter Py or Fe cylinder has a uniform state at remanence. The key point in the $\operatorname{Py}(42 \mathrm{~nm}) / 1 \mathrm{~nm} / \mathrm{Fe}(6 \mathrm{~nm})$ core-shell structure is the break of exchange and dipolar energy balance.

For larger nonmagnetic spacer thickness $(\eta>2.4 \mathrm{~nm})$, we have found a uniform Py core and an onion-like state in the $\mathrm{Fe}$ shell, with a pair of domain walls at the poles of the Py core. As shown in Fig. 5, this state comprises the magnetic patterns found in the isolated Py cylinder and the Fe shell, since the core and shell are out of reach of the dipolar field of each other.

\section{CONCLUSION}

Core-shell cylinders might be a promising starting point for key applications. Tailoring the vortex in the inner cylinder, or the domain-wall pair, in the shell, may be of interest for the design of high-density arrays for applications, such as vortex nano-oscillators [1], [2], or for the magnetic memories based on nanorings systems [3], [10], [11].

\section{ACKNOWLEDGMENT}

This work was supported in part by the Coordenação de Aperfeiçoamento de Pessoal de Nível Superior, in part by the Foundation for Research Support of Rio Grande do Norte, and in part by the Conselho Nacional de Desenvolvimento Científico e Tecnológico.

\section{REFERENCES}

[1] A. Dussaux et al., "Large microwave generation from current-driven magnetic vortex oscillators in magnetic tunnel junctions," Nature Commun., vol. 1, Apr. 2010, Art. ID 8.

[2] V. S. Pribiag et al., "Magnetic vortex oscillator driven by d.c. spin-polarized current," Nature Phys., vol. 3, pp. 498-503, May 2007.

[3] C. A. F. Vaz et al., "Ferromagnetic nanorings," J. Phys., Condens. Matter, vol. 19, no. 25, p. 255207, 2007.

[4] J. Rothman et al., "Observation of a bi-domain state and nucleation free switching in mesoscopic ring magnets," Phys. Rev. Lett., vol. 86, p. 1098 , Feb. 2001

[5] M. Kläui et al., "Domain wall pinning in narrow ferromagnetic ring structures probed by magnetoresistance measurements," Phys. Rev. Lett., vol. 90 , p. 097202, Mar. 2003.

[6] A. López-Ortega, M. Estrader, G. Salazar-Alvarez, A. G. Roca, and J. Nogués, "Applications of exchange coupled bi-magnetic hard/soft and soft/hard magnetic core/shell nanoparticles," Phys. Rep., vol. 553, pp. 1-32, Feb. 2015.

[7] M. Kläui, J. Rothman, L. Lopez-Diaz, C. A. F. Vaz, J. A. C. Bland, and Z. Cui, "Vortex circulation control in mesoscopic ring magnets," Appl. Phys. Lett., vol. 78, no. 21, p. 3268, 2001.

[8] C. M. Souza, A. L. Dantas, I. S. Queiroz, Jr., and A. S. Carriço, "Controlling the vortex core of thin permalloy nano-cylinders dipolar coupled to Co polarizers," J. Appl. Phys., vol. 115, no. 17, p. 17D110, 2014.

[9] R. W. Wang, D. L. Mills, E. E. Fullerton, J. E. Mattson, and S. D. Bader, "Surface spin-flop transition in $\mathrm{Fe} / \mathrm{Cr}(211)$ superlattices: Experiment and theory," Phys. Rev. Lett., vol. 72, p. 920, Feb. 1994.

[10] S. Jain and A. O. Adeyeye, "Aligned alternating head-to-head and tail-to-tail domain walls in ferromagnetic concentric rings," IEEE Trans. Magn., vol. 46, no. 6, pp. 1595-1598, Jun. 2010.

[11] C. B. Muratov and V. V. Osipov, "Bit storage by $360^{\circ}$ domain walls in ferromagnetic nanorings," IEEE Trans. Magn., vol. 45, no. 8, pp. 3207-3209, Aug. 2009. 